\title{
Philosophiques
}

\section{Précis de Lautman}

\section{Emmanuel Barot}

Volume 37, numéro 1, printemps 2010

Albert Lautman, philosophe des mathématiques

URI : https://id.erudit.org/iderudit/039719ar

DOI : https://doi.org/10.7202/039719ar

Aller au sommaire du numéro

Éditeur(s)

Société de philosophie du Québec

ISSN

0316-2923 (imprimé)

1492-1391 (numérique)

Découvrir la revue

Citer ce document

Barot, E. (2010). Précis de Lautman. Philosophiques, 37(1), 189-195.

https://doi.org/10.7202/039719ar

Ce document est protégé par la loi sur le droit d'auteur. L'utilisation des services d'Érudit (y compris la reproduction) est assujettie à sa politique d'utilisation que vous pouvez consulter en ligne.

https://apropos.erudit.org/fr/usagers/politique-dutilisation/ 


\title{
Disputatio \\ Précis de Lautman'
}

\author{
EMMANUEL BAROT \\ Université Toulouse-II Le Mirail
}

Le but de ce livre est d'introduire aux thèses principales de la philosophie mathématique d'Albert Lautman, dont la méconnaissance constituait naguère et constitue toujours une carence importante dans le champ de la philosophie et de l'épistémologie, francophones en particulier.

\section{Le rapprochement nécessaire de la métaphysique et des mathématiques}

La double singularité de Lautman, par le type de philosophie qu'il convoque, dont l'anachronisme semble la marque majeure, et par la technicité parfois très haute de ses exposés mathématiques, à la fois explique certainement cette méconnaissance, mais a contrario est tout aussi fortement stimulante, en raison notamment du fait, qui n'est pas anodin, de la légitimité qu'il a pu recueillir en son temps et ensuite chez un certain nombre de mathématiciens. Si l'argument d'autorité ne fait pas l'autorité de l'argument, cette reconnaissance m'a semblé encourager à la lecture de cette œuvre dense et foisonnante en raison des ouvertures et des reprises qu'elle a déjà ponctuellement suggérées, et dont on peut raisonnablement imaginer qu'elle est loin d'avoir livré toute sa fécondité. La volonté est donc double: ouvrir sur certaines pistes lautmaniennes à suivre aujourd'hui, comme prolongement naturel d'une introduction limitant l'exposé aux grandes thèses du penseur. Cependant l'ambition, d'une part, est d'illustrer suffisamment ces thèses pour qu'elles prennent corps sans que les détails n'en obscurcissent les significations majeures, mais d'autre part de ne faire l'impasse sur aucune de ces dernières, sans gommer indûment les tensions ou dimensions éventuellement aporétiques qui leur sont attachées.

Le fil conducteur du livre est l'affirmation de Lautman selon laquelle le «rapprochement de la métaphysique et des mathématiques n'est pas contingent, mais nécessaire ${ }^{2}$. L'exposition et l'interrogation des raisons et des voies de cette nécessité requiert l'articulation permanente entre les différents plans où elle prend sa source et s'atteste: ce qui implique, systématiquement, afin que les thèses majeures de Lautman prennent tout leur relief, d'exposer (1) les conditions et les significations du point de vue historique

1. Emmanuel Barot, Lautman, Paris, Les Belles-Lettres, Collection « Figures du Savoir », 2009 .

2. A. Lautman, «Nouvelles Recherches sur la structure dialectique des mathématique ", Les mathématiques, les Idées et le réel physique, Paris, Vrin, 2006, p. 237. 
de leur période d'énonciation, l'entre-deux-guerres français, et cela, dans le domaine des mathématiques comme dans celui de la philosophie, et (2) de restituer les problèmes théoriques spécifiques de chacun des deux champs (2a) dès lors qu'ils étaient ce par rapport ou en fonction de quoi Lautman a pris position ou a été orienté dans ses enquêtes, $(2 \mathrm{~b})$ ou réciproquement selon la portée ou l'impact qu'ont pu avoir les positions de Lautman sur leur traitement ultérieur.

D'où la progression retenue en quatre chapitres, dont les éléments essentiels sont les suivants.

Le premier chapitre "Les trois cadres de la pensée lautmanienne" est une sorte de seconde introduction détaillée, qui présente l'essentiel des révolutions, qui ont débuté au milieu du $\mathrm{XIX}^{\mathrm{e}}$ siècle, de l'analyse (son arithmétisation), de la géométrie (son algébrisation), et au niveau du développement de méthodes structurales pré-axiomatiques puis axiomatiques, en relation d'ailleurs avec la naissance de la nouvelle logique libérée de sa forme antérieurement aristotélicienne. C'est tout cela qui constitue la «matière» mathématique dont Lautman examine les formes les plus contemporaines, notamment celles des algébristes allemands et des futurs bourbakistes français.

En second lieu, c'est le point de vue de la métamathématique hilbertienne qui retient l'attention, puisque c'est exactement ce point de vue que Lautman entend reconduire, mais en l'investissant au travers d'une rupture qualitative avec le programme également hilbertien de réduction technique du problème des «fondements» de la mathématique. Lautman en défend au contraire une replatonisation, c'est-à-dire la ré-assimilation de cette métamathématique à une dialectique de type métaphysique selon une perspective (néo)platonicienne plutôt complexe. L'idée est, dès ce moment du livre, d'indiquer que la philosophie lautmanienne va rapidement se débattre avec les difficultés attachées aux tentations «régaliennes» des métaphysiques qui, jadis, prétendaient être le lieu de saisie et de formulation des principes d'une science mathématique posée comme inapte, en elle-même et par elle-même, à s'en charger.

Enfin est présenté le troisième "cadre ", celui des épistémologies à la fois historicisantes et «dialectiques» ou «dialectisantes» de l'entre-deuxguerres dans lequel il intervient. Par là, Lautman est clairement réinscrit dans un milieu intellectuel dont les membres partagent un ensemble assez net de soucis et d'interrogations. Avec Bachelard, Cavaillès, ou encore Gonseth, il s'efforce de rendre raison de l'objectivité et de la nécessité des mathématiques tout en assumant leur historicité et la contingence des découvertes ou crises qu'elles rencontrent. De même s'efforce-t-il de mettre en valeur le dynamisme, la temporalité de cette connaissance contre sa réduction plus ou moins fixiste et désincarnée, et d'identifier le type d'objets et d'objectivité irréductibles qui sont les siennes contre leur rabattement sur des propriétés 
logico-syntaxiques des langages formels: autant de préoccupations convergeant à la fois contre les orientations pré-historicistes - par exemple, de la tradition transcendantale kantienne et néo-kantienne dans le premier cas contre le développement conquérant du néo-positivisme, entre empirisme radical et logicisme dans le second.

Cette triple réinscription permet de montrer que la philosophie de Lautman n'est pas, loin s'en faut, un îlot incommensurablement isolé. Mais elle rend du même mouvement possible la mise en lumière, en un second temps, de la posture décentrée que Lautman va développer, notamment visà-vis de ses coréligionnaires dialectisants.

\section{II}

L'objectif de ce premier chapitre était de préparer le terrain à l'exposé en deux temps, ou plutôt en deux "strates", qui constitue l'essentiel du second chapitre "Du réel en mathématiques». On y expose, en en laissant de côté les apories, en le prenant pour "acquis" si l'on peut dire, le rapport générique établi par Lautman entre théories mathématiques d'un côté et Idées et notions métaphysiques (dialectiques) de l'autre, rapport qui s'énonce comme différence ontologique entre deux plans de ce qui fait la « réalité » des mathématiques, de ce qu'elles sont et de ce qu'elles prennent en charge. Ce rapport s'éclaire chez Lautman à travers son enquête sur le caractère radicalement non monolithique de la "réalité » mathématique. Dominé par le souci platonicien de saisir son unité au travers de sa multiplicité, il enregistre et questionne les quatre formes majeures qu'elle prend: des "faits " (découvertes, ruptures, etc.) qui scandent l'histoire aux «Idées » qui en gouvernent l'organisation synchronique et diachronique, en passant par les «théories » dont il examine alors analogies structurelles et affinités méthodologiques, à partir et au-delà des différents domaines sémantiques, champs d'objectivité, bref, des types ou classes d' «êtres» qui les peuplent.

Pour Lautman le processus historique de la mathématique est un processus d'incarnation, de réalisations successives de grandes Idées, c'est-àdire de quelques grands couples de notions qui constituent à ses yeux de véritables "problèmes» ou énigmes de la rationalité connaissante depuis l'Antiquité. Les exemples pris dans le livre, choisis dans des champs mathématiques variés (le couple structure-existence en logique mathématique, le couple intrinsèque-extrinsèque en topologie, le couple continu-discontinu en analyse et en arithmétique, etc.), servent ainsi à montrer de façon détaillée comment des domaines mathématique différents «incarnent» pour lui une même Idée, et réciproquement, comment une même Idée se pluralise lorsqu'elle «passe » au plan des théories. C'est par ces allers-retours répétés de la multiplicité à l'unité que Lautman prétend capter «l'unité des mathématiques dans leur développement actuel ", pour reprendre le titre d'une de ses deux thèses de 1937, c'est-à-dire capter la nature et les ressorts dynamiques de la productivité mathématique au cours de son développement historique, sur 
la base des effets produits par ces décennies révolutionnaires antérieures dont il tire les leçons.

Par là est exposé à un premier niveau, relativement schématisé, le rapport ontologie/histoire au cœur du dispositif, à partir des grandes lignes d'interprétation reprises par Lautman, et en particulier l'opposition entre la mathématique "classique ", et la mathématique "moderne» qui est avant tout marquée du sceau de l'esprit et des méthodes algébriques.

\section{III}

Le second chapitre visait avant tout à illustrer la façon dont Lautman procède dans son œuvre pour présenter et interpréter le texte de la mathématique, et ce qui ressort de cette interprétation, et à fournir un premier cadre de présentation du problème philosophique de l'articulation métaphysique/ histoire. Le troisième chapitre "La différence ontologique face à l'histoire » est donc consacré, en détail cette fois, aux difficultés proprement philosophiques soulevées par cette «différence ontologique» et ces «Idées » qu'il reprend à Platon, et en partie au Platon "ésotérique », celui de la doctrine orale des premiers principes indirectement transmise par la Métaphysique d'Aristote. La nature hautement problématique de ces Idées tient à leur "dialectique» spécifique qu'il reformule pour partie avec la distinction de Heidegger entre Être et étants, mais selon une tournure qui semble fortement apparentée avec le processus d'actualisation de l'Idée tel que Hegel le présente dans sa Science de la logique. D'où les confrontations successives de Lautman avec ces figures, en particulier celle avec Hegel qui n'allait pas de soi puisque Lautman ne le cite jamais.

Le bilan de ces rapprochements est finalement assez simple: Lautman est un parfait hétérodoxe qui se saisit, chez une autorité quelconque, de motifs théoriques qui entrent en résonance avec les mathématiques dans lesquelles il baigne, et en raison de cette résonance, mais au travers d' "infidélités » et de torsions infligées avec une grande liberté aux usages ou définitions d'origine des concepts ou opérateurs théoriques en question. Le résultat est qu'il n'est ni heideggerien, ni hégélien, ni platonicien non plus, mais plutôt promoteur d'une métaphysique d'esprit platonicien qui l'apparente jusqu'à un certain point, mais un certain point seulement, à certains traits hégéliens, en particulier du fait de la thèse selon laquelle l'Idée dialectique, par elle-même transcendante, ne peut pourtant s'incarner, se réaliser et surtout se déterminer — sans jouer sur les mots, trouver à la fois "complétude» et «consistance» — que par la médiation historique de la théorisation et de la formalisation, c'est-à-dire dans l'immanence des pratiques mathématiciennes historiquement situées.

Schématiquement résumé, on peut dire que pour Lautman l'histoire est le lieu ou le plan de résolution de problèmes non historiques par euxmêmes, problèmes qui ne sont pourtant jamais épuisables par les théories dans leurs développements et successions, justement parce qu'en leur ordre 
propre, ces «problèmes» sont «indéterminés ", c'est-à-dire ouverts par principe à une multiplicité, a priori sans limite, de traductions. Bref, l'histoire est une réponse continuée et sans fin à des énigmes irréductibles et insolvables comme telles. Telle est pour Lautman la loi du progrès mathématique, ce qui fait son unité profonde, "organique», son unité vitale, à l'intérieur comme au-delà de la diversité des champs, domaines d'objets, et méthodes qui en font toute l'exponentielle richesse. Son insistance sur ce plan ontologico-métaphysique, son affirmation réitérée selon laquelle ce dernier n'est pas soluble dans l'historicité des théories, est très exactement ce par quoi Lautman impose sa singularité et se décentre des épistémologies principalement historicistes de ses congénères, et de leur maître commun qu'avait été Brunschvicg.

\section{IV}

Le quatrième chapitre "Une cosmologique physique sous tension» cherche, enfin, à clarifier le chantier partiellement distinct de cette nouvelle "philosophie de la physique» que Lautman a progressivement défriché. À la dualité Idées/théories se rajoute alors un troisième pôle, celui des phénomènes naturels objectivés en physique. L'orientation principale de Lautman en ce domaine ne surprend pas: de même que les Idées "dominent» les théories, elles dominent les phénomènes. C'est là que ses rapports avec le kantisme s'éclairent: derrière la reprise partielle de formules issues de la philosophie transcendantale, c'est bien le modèle anti-kantien du Timée qui reste déterminant, avec lequel la confrontation était donc indispensable. Sur le temps, tout particulièrement, Lautman développe l'idée selon laquelle la distinction entre le "paramètre indépendant» de la mécanique classique et la "quatrième dimension» du continuum relativiste est l'expression physique d'une dualité proprement, et de façon logiquement antérieure, mathématique, qui n'est elle-même que l'expression de la dualité dialectique entre le "Même» et l'«Autre» platoniciens. Cette dérivation du métaphysique au phénoménal trouve d'ailleurs aussi sa formulation, dans le Timée, dans la création démiurgique de l'univers sensible (spatio-temporel) du devenir, qui est en son principe le résultat d'une composition de figures mathématiques, les polyèdres réguliers.

On présente là encore d'autres exemples de «situations » illustrant cette triade hiérarchisée phénomène-théorie-Idées afin de montrer la transversalité de la thèse lautmanienne. Par exemple son interprétation de la répartition des fonctions d'onde en symétriques et en dissymétriques, par lesquelles sont modélisées les trajectoires respectives de particules élémentaires selon les caractéristiques du système physique considéré, qui repose pour lui sur le nombre pair ou impair de leurs constituants. Ce qui l'amène à dire le fondement même du rapport entre le phénoménal et le physique relève des propriétés du pair et de l'impair en théorie des permutations. Mais comme ces propriétés sont expressives de la domination du couple 
symétrique-dissymétrique qui est l'une des Idées dialectiques principales, c'est bien là encore la dualité Même-Autre (laquelle n'a elle-même qu'un visage de la dualité originelle entre l'Un et la dyade) qui apparait comme fondamentale.

Rien n'empêche d'imaginer que cette cosmologie "qualitative", très certainement en porte-à-faux avec les idées dominantes en la matière, ne puisse constituer aujourd'hui un authentique et stimulant "programme métaphysique de recherche».

\section{La leçon de Lautman}

En conclusion on s'efforce d'articuler les deux types de réception, mathématique et philosophique de cette œuvre en se concentrant sur quelques-uns de leurs lieux majeurs ou figures principales. On rappelle synthétiquement d'une part que G. Deleuze dans Différence et répétition fut le premier et seul véritable "passeur" de Lautman dans la philosophie de l'après-guerre, et que son geste fut reconduit dans la seconde moitié des années 1980 par J. Petitot et J.-M. Salanskis. Les accents phénoménologiques-transcendantaux et herméneutiques qui dominent les lectures de ces derniers, manifestement en décalage partiel avec la lettre de l'œuvre, ont néanmoins marqué, dès lors, que cette œuvre est susceptible de réappropriations et de poursuites fécondes attestant l'ampleur de ses vues.

La réception mathématique, quant à elle, est présentée en deux temps. Après avoir souligné la proximité d'esprit et d'orientations, la communauté d'objets et de préoccupations de Lautman avec la génération fondatrice de Bourbaki, on souligne les radicales divergences conceptuelles qui s'attachent à leurs lectures respectives du sens de cette expérience mathématique: le réalisme mathématique des seconds et le "réalisme" métaphysique des Idées du premier ont en effet peu en commun. C'est justement ce hiatus que des héritiers comme C. Ehresmann ont ultérieurement suggéré de résorber en esquissant une «traduction» du motif principal de la dualité, qui est au cœur du dynamisme des Idées, dans les termes d'un idiome mathématique à la fois transversal et spécifique, celui des catégories. Raison pour laquelle on met alors essentiellement l'accent sur le problème de la «formalisation» des Idées que F. Zalamea, dans le sillage d'Ehresmann, a récemment réactualisé. Ce projet constitue en effet un point d'ancrage majeur de tout examen renouvelé de la "nécessité » qui lie mathématiques et philosophie, c'est-à-dire de toute tentative pour en ré-identifier aujourd'hui les contours respectifs, les "territoires", et donc les types, modes et lieux, qu'ils soient propices à de fortes tensions ou non, de possibles collaborations.

Parasiter ainsi les partages disciplinaires, promouvoir une stratégie interprétative de ces champs trop souvent indûment réifiés et figés dans leurs univers respectifs, mobiliser à cette fin des schèmes dialectiques qui sont par tradition et par définition des schèmes de la médiation et d'une compréhension adéquate, contre tout relativisme et tout fixisme à la fois, de la réalité 
dynamique du multiple, telle est la grande leçon de Lautman. Cette leçon l'extrait de façon emblématique des problématiques souvent étroitement confinées de la discipline des «fondements» de la logique et des mathématiques, et l'impose, en toute son ampleur, comme un protagoniste d'envergure du dialogue millénaire nouant la science la plus «pure » et la métaphysique la plus ambitieuse. Par la liberté dont Lautman a témoigné à la fois théoriquement et pratiquement dans son combat général contre la tyrannie du fait et la soumission à l'établi, il ressort en ce sens comme un grand «classique», si l'on veut bien entendre par là non pas un penseur du passé et/ou dépassé, mais un penseur qui s'est mis à la hauteur des interrogations et des enjeux qui lui faisaient face, dans leur globalité, dans leur substance énigmatique, et même dans leur beauté. À mes yeux, nul doute que dans cette posture réside l'un des facteurs majeurs de son isolement, mais tout autant l'un des puissants motifs pour sa re-lecture aujourd'hui.

Qu'on partage ou non les thèses de Lautman est tout à fait secondaire par rapport au sens des enjeux dont il fait preuve dans la façon dont il traite ce qu'il se donne comme objet. Contribuer à la diffusion de sa posture, indépendamment même de la littéralité de ses positions, est en tous cas l'objectif majeur du livre, et ce qui a constitué dès le départ sa principale motivation. 